\title{
Isogeometric Simulation of Turbine Blades for Aircraft Engines
}

\author{
David Großmann ${ }^{1}$, Bert Jüttler ${ }^{2}$, Helena Schlusnus ${ }^{3}$, Johannes Barner ${ }^{1}$, Anh-Vu Vuong ${ }^{4}$ \\ ${ }^{1}$ MTU Aero Engines GmbH, Munich, Germany \\ 2 Johannes Kepler University, Institute of Applied Geometry, Linz, Austria \\ 3 Technical University, Centre for Mathematical Sciences, Munich, Germany \\ 4 Technical University, Centre for Applied Mathematics, Kaiserslautern, Germany
}

\begin{abstract}
Isogeometric analysis is a novel approach to numerical simulation that has the potential to bridge the gap between geometric design and numerical analysis. It uses the same exact geometry representation in all stages of the product development. In this paper we present recent results which demonstrate the competitiveness of the new concept in an industrial environment, more precisely, in the challenging field of aircraft engines. We study the deformation of turbine blades under the assumption of linear elasticity by considering all major loads and boundary conditions of a standard mechanical simulation process for turbine blades. We use the numerical approximations obtained by the classical finite element method as a benchmark for the capabilities of the new concept. It is shown that it is able to reach comparable results using only a small fraction of the number of degrees of freedom required by the classical method. Thus, isogeometric analysis allows using much coarser geometric representations for numerical simulation.
\end{abstract}

\section{Introduction}

The commercial activities of the MTU Aero Engines focus on developing, manufacturing and repairing turbine engines for aircrafts. A challenging task in this field is the efficient interaction between the geometric design and the numerical simulation of the individual components. In general, a designer generates a CAD (computer aided design) model which has to be prepared and transformed into a mesh for the finite element analysis (FEA). This process is still a bottleneck in the overall engineering process.

An impressive and promising concept to bridge this gap is referred to as isogeometric analysis (IGA) which was introduced in [19]. It uses the geometric representation of the object, parameterized by B-splines or NURBS as the CAD standard, to define the finite-dimensional spaces needed for the Galerkin projection within the numerical analysis. As a major advantage, only one representation of the object is required and consequently the geometrical errors introduced by the approximation of the physical domain by a computational mesh in the finite element method (FEM) are eliminated. Furthermore a significant reduction of the number of unknowns and an efficient optimization process becomes possible.

Since its introduction in 2005, IGA has attracted enormous interest from the academic community, resulting in a substantial number of related publications. These publications can be organized in four groups.

First, IGA has been successfully applied to various specific simulation problems, including linear elasticity, simulation in electromagnetics, and flow simulations, e.g. $[8,14,26]$. These publications clearly demonstrate the applicability for large classes of problems and often the superiority of the new concept compared to the standard finite element approaches.

Second, several authors have studied several theoretical aspects of IGA, such as error estimates, convergence rates, stability issues, and numerical quadrature rules, e.g. in $[3,5,22,28]$. It was shown that many of the theoretical results obtained for the classical finite element analysis can be generalized to the isogeometric case.

The third group of publications deals with the nontrivial problem of domain parameterization for IGA. Suitable parameterizations can be obtained either by directly designing them for specific applications or by deriving them from an existing CAD model or from a triangular mesh representation [1, 9, 16, 24]. Volumetric representations such as polycube splines [31] may be useful in the future.

Fourth, the use of tensor-product spline spaces in IGA triggered new research on generalizations of these spaces providing local adaptivity. Several concepts are currently being developed in parallel, including T-splines, hierarchical splines, and polynomial splines over hierarchical T-meshes [4, 6, 10, 11, 13, 23, 25, 30].

Based on these and other very promising results from the academic community, we demonstrate the capabilities of the new concept of IGA in a real-world industrial environment. More precisely, we will use it for the numerical simulation of turbine blades as one of the crucial steps in the design of highly efficient turbine engines. Based on a given volumetric B-spline parameterization of turbine blades we study their deformation under centrifugal forces and mechanical and thermal loads given by the aerodynamical and thermodynamical analysis. For the sake of brevity we shall denote both turbine blades and compressor blades as turbine blades or blades only.

After a brief introduction to the volumetric turbine blade model and the governing equations and variables of linear elasticity we use the isogeometric concept to derive a system of linear equations determining the displacement field. With the focus on the industrial applicability, we present simulation examples incorporating the required boundary conditions and compare them with the finite element calculations.

\section{Turbine blade model}

We introduced a framework to model a single trivariate B-spline volume of a turbine blade with the focus on the industrial applicability in [16]. Starting from a triangular mesh of a blade, generated by an optical measurement system or by sampling a standard (surface) CAD description, a single-patch volumetric parameterization

$$
\mathbf{F}\left(\xi_{1}, \xi_{2}, \xi_{3}\right)=\sum_{\substack{j_{1}=1, \ldots, n_{1} \\ j_{2}=1, \ldots, n_{2} \\ j_{3}=1, \ldots, n_{3}}} N_{j_{1}}\left(\xi_{1}\right) N_{j_{2}}\left(\xi_{2}\right) N_{j_{3}}\left(\xi_{3}\right) \mathbf{d}_{j_{1}, j_{2}, j_{3}}
$$

with the domain $\boldsymbol{\Omega}_{0}=[0,1]^{3}$ was constructed by a semiautomatic process. The univariate B-spline basis functions $N_{j_{1}}\left(\xi_{1}\right), N_{j_{2}}\left(\xi_{2}\right)$ and $N_{j_{3}}\left(\xi_{3}\right)$ of a certain degree $d$ are defined with respect to three given knot vectors $\Xi_{1}, \Xi_{2}$ and $\Xi_{3}$ with degree-fold boundary knots. The vector-valued 


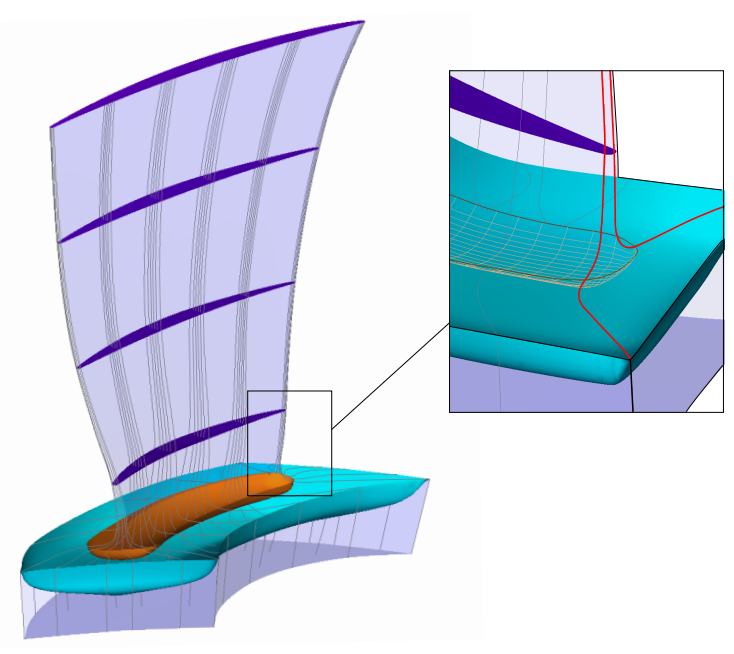

Figure 1: The figure shows several parametric curves and surfaces of a trivariate B-spline model of a turbine blade. The airfoil and the block part are two $C^{0}$ connected B-spline volumes. The singular curves of the parameterization coincide with the transition between the edges and the sides of the airfoil, called wedge curves (shown in red).

coefficients $\mathbf{d}_{j_{1}, j_{2}, j_{3}} \in \mathbb{R}^{3}$ are called control points. See [17] for more information on B-spline techniques.

The resulting volumetric model is visualized in Figure 1. It supports all standard blade features such as

- faithful representation of wedge curves,

- representation of leading and trailing edge,

- reproduction of the fillet curve, and

- the natural flow of the blade at the fillet,

see [16] for more information.

An additional block represents a part of the disk and is connected by the $d$-fold knot $\xi_{3}=\bar{\xi}_{3}$. The choice of $\mathrm{B}$-splines (or the more general NURBS) ensures the compatibility to the standard CAD and CAE systems.

Now, the so-called geometry function $\mathbf{F}$ (i.e., the volumetric parameterization) enables us to explore the new numerical simulation approach of isogeometric analysis. In addition, a fully automatic structured mesh generation for the standard finite element method becomes available: A discretization of $\xi_{1}, \xi_{2}$ and $\xi_{3}$ in the parameter space $\boldsymbol{\Omega}_{\mathbf{0}}$ generates nodes and hexahedral elements of a structured mesh in the physical space $\boldsymbol{\Omega}$. We use quadratic hexahedral elements for the finite element simulations in the presented work, which were performed to compare the results with isogeometric analysis. The singular hexahedral elements along the wedge curves are replaced by pairs of quadratic wedge elements.

\section{Linear elasticity}

We consider a three-dimensional solid $\Omega \subset \mathbb{R}^{3}$ consisting of a polycrystalline material for which an isotropic and linear elastic behavior is assumed. The solid $\boldsymbol{\Omega}$ is deformed under some prescribed mechanical and thermal loads which are constant over time: The volume forces $\hat{\mathbf{b}}=\left(\hat{b}_{1}, \hat{b}_{2}, \hat{b}_{3}\right)^{T}$, the boundary tractions $\hat{\mathbf{t}}=\left(\hat{t}_{1}, \hat{t}_{2}, \hat{t}_{3}\right)^{T}$, the boundary displacements $\hat{\mathbf{u}}=\left(\hat{u}_{1}, \hat{u}_{2}, \hat{u}_{3}\right)^{T}$ and the thermal expansion caused by the inhomogeneous temperature distribution within $\boldsymbol{\Omega}$. These loads cause a change of the shape of the solid denoted by the (unknown) displacement field $\mathbf{u}=\left(u_{1}, u_{2}, u_{3}\right)^{T}$.
We use the index notation for the following vector and tensor operations. The subscripts are 1,2,3 and refer to the three-dimensional vectors and vector fields unless it is stated otherwise. The one-dimensional components $\Omega$ and $\Gamma$ of the three-dimensional domains $\boldsymbol{\Omega}$ and $\boldsymbol{\Gamma}$ are denoted without subscripts for the sake of readability.

The mechanical situation at a material point of the solid under the prescribed loads and boundary conditions is described by the following strong form of the boundary value problem: Find the displacements $u_{i}: \bar{\Omega} \rightarrow \mathbb{R}$ $(i=1,2,3)$ such that

$$
\begin{aligned}
\frac{\partial \sigma_{i j}}{\partial x_{j}}+\hat{b}_{i} & =0 & & \text { in } \Omega, \\
\frac{1}{2}\left(\frac{\partial u_{i}}{\partial x_{j}}+\frac{\partial u_{j}}{\partial x_{i}}\right) & =\varepsilon_{i j} & & \text { in } \Omega, \\
c_{i j k l}\left(\varepsilon_{k l}-\alpha \Delta T \delta_{k l}\right) & =\sigma_{i j} & & \text { in } \Omega, \\
u_{i} & =\hat{u}_{i} & & \text { on } \Gamma_{D}, \\
\sigma_{i j} n_{j} & =\hat{t}_{i} & & \text { on } \Gamma_{N},
\end{aligned}
$$

with $\Gamma_{D} \cup \Gamma_{N}=\Gamma=\partial \Omega$ for the given Dirichlet boundary conditions (5) and the Neumann boundary conditions (6) with the unit outward normal $\mathbf{n}=\left(n_{1}, n_{2}, n_{3}\right)^{T}$. The vector $\mathbf{x}=\left(x_{1}, x_{2}, x_{3}\right)^{T}$ denotes the spatial components of the Cartesian coordinate system in the physical space.

The equilibrium equation (2) relates the (symmetric) stress tensor $\sigma_{i j}$ to the given volume forces $\hat{b}_{i}$. The strain tensor $\varepsilon_{i j}$ is defined to be the symmetric part of the displacement gradient under the assumption of small displacements (3). The constitutive equation (4) relates the stress tensor to the strain tensor by the elastic coefficients $c_{i j k l}$,

$$
c_{i j k l}=\lambda \delta_{i j} \delta_{k l}+\mu\left(\delta_{i k} \delta_{j l}+\delta_{i l} \delta_{j k}\right),
$$

where $\delta_{i j}$ denotes the Kronecker delta. The Lamé parameters $\lambda$ and $\mu$ are often expressed by the Young's modulus $E$ and Poisson's ratio $\nu$

$$
\lambda=\frac{\nu E}{(1+\nu)(1-2 \nu)} \quad \text { and } \quad \mu=\frac{E}{2(1+\nu)} .
$$

The thermal expansion coefficient $\alpha$ of a material quantifies the change in volume with a change in temperature.

It is extremely important to model the influence of the temperature on the material properties in the blade design, since the turbine blades reach their final shape only under the influence of temperature, centrifugal forces and pressure fields.

See $[7,18]$ for more information on linear elasticity.

\section{Isogeometric analysis}

Now, we outline the main features of the isogeometric concept and apply them to the derived boundary value problem of linear elasticity for three-dimensional solids. For more information on isogeometric analysis, see [19, 20], and especially for the simulation of solids, see [1, 29].

\subsection{Weak form}

The classical finite element concept and the new isogeometric concept are both based on the weak form as a variational formulation of the strong form (2)-(6). The weak form requires two function spaces.

First, the space $\mathcal{V}$

$$
\mathcal{V}=\left\{\mathbf{v}=\left(v_{1}, v_{2}, v_{3}\right)^{T}\left|v_{i} \in \mathcal{H}^{1}(\Omega), v_{i}\right|_{\Gamma_{D}}=0\right\}
$$


consists of all test functions $\mathbf{v}$ that vanish on the Dirichlet boundary of $\boldsymbol{\Omega}$. Second, the solution space $\mathcal{S}$,

$$
\mathcal{S}=\left\{\mathbf{u}=\left(u_{1}, u_{2}, u_{3}\right)^{T}\left|u_{i} \in \mathcal{H}^{1}(\Omega), u_{i}\right|_{\Gamma_{D}}=g\right\},
$$

contains all potential solution functions $\mathbf{u}$ which are required to satisfy the Dirichlet boundary conditions (5). The Sobolev space $\mathcal{H}^{1}(\Omega)$ consists of all functions on $\Omega$ with weak and square-integrable first derivatives.

We obtain the variational formulation of the strong form by multiplying the equilibrium equation (2) and the Neumann boundary conditions (6) with arbitrary test functions (9) and by integrating over $\boldsymbol{\Omega}$. The resulting weak form of the problem is stated as follows: For given volume forces $\hat{b}_{i}$, surface tractions $\hat{t}_{i}$ and boundary displacements $\hat{u}_{i}$, find $\mathbf{u} \in \mathcal{S}$ such that for all $\mathbf{v} \in \mathcal{V}$,

$$
\int_{\boldsymbol{\Omega}} \sigma_{i j} \frac{\partial v_{i}}{\partial x_{j}} \mathrm{~d} \mathbf{x}=\int_{\boldsymbol{\Omega}} \hat{b}_{i} v_{i} \mathrm{~d} \mathbf{x}+\int_{\boldsymbol{\Gamma}_{N}} \hat{t}_{i} v_{i} \mathrm{~d} \mathbf{x} .
$$

Replacing the partial differential equations of equilibrium in the strong form by an integral in the weak form leads to a formulation which is more suitable for finite element analysis and isogeometric analysis.

The choice of the test functions in the weak form is arbitrary and we look upon $v_{i}$ as the virtual displacements $v_{i}=\delta u_{i}$. Inserting equations (3) and (4) into the weak form results in the principle of virtual work

$$
\begin{array}{r}
\int_{\Omega} c_{i j k l} \frac{\partial \delta u_{i}}{\partial x_{j}}\left(\frac{\partial u_{k}}{\partial x_{l}}-\alpha \Delta T \delta_{k l}\right) \mathrm{d} \mathbf{x} \\
=\int_{\Omega} \hat{b}_{i} \delta u_{i} \mathrm{~d} \mathbf{x}+\int_{\Gamma_{N}} \hat{t}_{i} \delta u_{i} \mathrm{~d} \mathbf{x} .
\end{array}
$$

Equation (12) is still a continuous formulation of the problem and we have to transform it to a discrete formulation to solve it numerically.

\subsection{Galerkin projection}

The Galerkin projection is the most famous approach to project the continuous problem stated by the weak form (11) into a discrete one by replacing the function spaces $\mathcal{S}, \mathcal{V}$ with finite dimensional subspaces $\mathcal{S}^{h} \subset \mathcal{S}, \mathcal{V}^{h} \subset \mathcal{V}$ where $h$ indicates the discretization of the domain $\boldsymbol{\Omega}$. The function $\mathbf{u}^{h} \in \mathcal{S}^{h}$ is constructed as a linear combination

$$
\mathbf{u}^{h}=\phi_{0}+\sum_{j=1}^{n} \phi_{j} \mathbf{q}_{j}
$$

with coefficients $\mathbf{q}_{j}$ and basis functions $\phi_{j}: \boldsymbol{\Omega}_{0} \rightarrow \mathbb{R}$ for $j=1, \ldots, n$. The function $\phi_{0}$ fulfills the Dirichlet boundary conditions. The key point is to observe that $\mathcal{S}^{h}$ and $\mathcal{V}^{h}$ are composed of identical collections of functions. In the classical finite element approach, the subspace $\mathcal{V}^{h}$ consists of piecewise $C^{0}$ connected polynomials. As a result, the given geometry $\mathbf{F}$ is approximated by the linear combination of the basis functions $\phi_{j}$ and the three-dimensional nodes of the generated finite element mesh. In most practical applications, low order polynomials are used as basis functions. We choose a triquadratic polynomial basis for the finite element simulations in the present work.

\subsection{Isogeometric approach}

The isogeometric approach takes the exact geometry function $\mathbf{F}$ into account and uses the spanned function space to define the solution and weighting space $\mathcal{S}^{h}, \mathcal{V}^{h}$. In the present work, the turbine blades are modeled by a single trivariate B-spline volume (1). We rewrite the geometry function $\mathbf{F}: \boldsymbol{\Omega}_{0} \rightarrow \mathbb{R}^{3}$ as

$$
\mathbf{F}\left(\xi_{1}, \xi_{2}, \xi_{3}\right)=\bar{N}_{j}\left(\xi_{1}, \xi_{2}, \xi_{3}\right) \mathbf{d}_{j},
$$

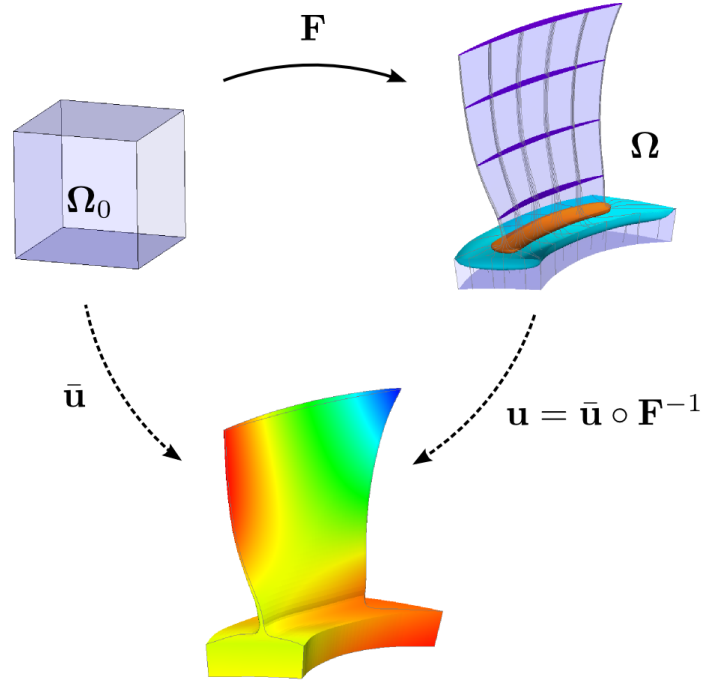

Figure 2: In the isogeometric concept, the finite-dimensional space for the Galerkin projection is obtained by composing the B-spline (resp. NURBS) basis functions $\overline{\mathbf{u}}$ with the inverse of the geometry parameterization $\mathbf{F}$.

where we use the Einstein summation convention with respect to the index $j$. More precisely, we assume a lexicographical order of the control points $\mathbf{d}_{j}=\left(d_{1 j}, d_{2 j}, d_{3 j}\right)^{T} \in$ $\mathbb{R}^{3}$ with $j=1, \ldots, \bar{n}$ for $\bar{n}=n_{1} n_{2} n_{3}$.

The basis functions $\bar{N}_{j}$ are products of three univariate B-splines with the three parameters $\xi_{1}, \xi_{2}, \xi_{3}$.

Following the isogeometric idea, the basis functions $\phi_{1}, \ldots, \phi_{n}$ which are used for the Galerkin projection are defined by the same (B-spline) basis functions as for the geometry functions $\mathbf{F}=\left(F_{1}, F_{2}, F_{3}\right)$ in (14). The knot insertion property of $\mathrm{B}$-splines provides a simple and fast refinement operator for the analysis part that leaves the geometry and the mapping between $\boldsymbol{\Omega}_{0}$ and $\boldsymbol{\Omega}$ invariant. Unfortunately, knot insertion has a global refinement effect due to the tensor product structure of B-spline manifolds. For recent work on local refinement strategies, see [27, 30]. For a theoretical study of the effect of global refinement techniques, see [5].

To define the approximate displacement functions $u_{i}^{h}: \Omega \rightarrow \mathbb{R}$ in the physical space (where the index $i=$ $1,2,3$ indicates the space coordinate), which represent the numerically obtained approximate solution, the functions $\bar{u}_{i}^{h}$ are "pushed forward" by the geometry function,

$$
u_{i}^{h}=\bar{u}_{i}^{h} \circ \mathbf{F}^{-1}=\bar{N}_{j}\left(\mathbf{F}^{-1}\left(x_{1}, x_{2}, x_{3}\right)\right) q_{i j},
$$

see Figure 2. The boundary conditions are included into (15) by pre-assigning suitable values to some of the control variables $q_{i j}$.

Thus, the finite dimensional subspace $\mathcal{V}^{h}$ is a subspace of the pushed-forward basis functions,

$$
\mathcal{V}^{h} \subset \operatorname{span}\left\{\bar{N}_{j} \circ \mathbf{F}^{-1}\right\}_{j=1, \ldots, \bar{n}}
$$

Inserting the isogeometric projection (15) into the principle of virtual work (12) leads to the linear system,

$$
\mathbf{A q}=\mathbf{b}
$$

with the stiffness matrix $\mathbf{A} \in \mathbb{R}^{3 \bar{n} \times 3 \bar{n}}$ and the load vector 
$\mathbf{b} \in \mathbb{R}^{3 \bar{n}}$

$$
\begin{aligned}
\mathbf{A}_{i m, k n} & =\int_{\boldsymbol{\Omega}_{0}}\left(\frac{\partial \bar{N}_{m}}{\partial \xi_{h}} \frac{\partial \xi_{h}}{\partial x_{j}}\right)\left(\frac{\partial \bar{N}_{n}}{\partial \xi_{h}} \frac{\partial \xi_{h}}{\partial x_{l}}\right) c_{i j k l} J \mathrm{~d} \boldsymbol{\xi} \\
\mathbf{b}_{i m} & =\int_{\boldsymbol{\Omega}_{0}}\left(\hat{b}_{i} \bar{N}_{m}+\frac{\partial \bar{N}_{m}}{\partial \xi_{h}} \frac{\partial \xi_{h}}{\partial x_{j}} c_{i j k l} \alpha \Delta T \delta_{k l}\right) J \mathrm{~d} \boldsymbol{\xi} \\
& +\int_{\boldsymbol{\Gamma}_{N_{0}}} \hat{t}_{i} \bar{N}_{m} \mathrm{nd} \boldsymbol{\xi}
\end{aligned}
$$

for $n, m=1, \ldots, \bar{n}$ and $i, k=1,2,3$, where the symbols $c_{i j k l}, \hat{b}_{i}$ and $\hat{t}_{i}$ represent the elastic coefficients, the volume forces and the boundary tractions, respectively.

Note that the integrals are defined over the parametric domain $\boldsymbol{\Omega}_{0}$ and therefore the substitution rule was applied, involving the Jacobian matrix $\mathbf{J}_{\mathbf{F}}$ and its determinant $J=\left|\operatorname{det} \mathbf{J}_{\mathbf{F}}\right|$. Moreover, $\mathrm{n}=|\mathbf{n}|$ for the surface normal $\mathbf{n}$ of the parameterized boundary surface.

Due to the properties of B-splines and the partial differential equation stated in the weak form (11), the matrix $\mathbf{A}$ is symmetric, positive definite and sparse and the unknown control variables $q_{i j}$ are well-defined. The integrals are approximately evaluated by Gaussian quadrature (with four points for each knot span), see [2,21] for a discussion of integration methods for the isogeometric approach.

We conclude this section with some remarks on the isogeometric concept:

- Instead of using two different models, the geometry and the analysis model, the isogeometric approach is based on only one model with an exact representation of the geometry. Therefore, the non-trivial task of generating a finite element mesh is no longer required. The exact geometry is employed at all levels of discretization, even in the analysis part, by the available refinement operations, particularly knot insertion and degree elevation. However, the geometry parameterization has to be "analysis suitable", cf. [9].

- Using B-splines (respectively NURBS) as basis functions instead of piecewise $C^{0}$ connected polynomials leads to a higher and controllable continuity and to a larger support of the basis functions. Therefore, the basis does not interpolate the control points and variables. As a consequence one can interpret these quantities only in combination with the related basis functions. In addition, the incorporation of the boundary conditions is not as simple as in the finite element concept. We will choose a least-squares fit of the given Dirichlet boundary conditions to define the related control variables. See [20,30] for more information on this challenging topic.

- Due to the tensor-product structure of higherdimensional B-spline (respectively NURBS) functions, the parameterization of non-quadrangular domains leads to singularities. Especially for singlepatch parameterizations, such as the given turbine blade model, the edges of the physical domain are often singular. This does not affect the calculation of the displacements because we evaluate the integrals in the linear system (17) only at the Gauss nodes which does not use the ends of the parameter interval. The calculation of the strain (3) in the postprocessing uses the derivatives of the displacements with respect to the coordinates $x_{i}$ which are collinear along the singular parameter directions. This problem occurs also in the finite element method and we overcome it by using slightly perturbed parameters to evaluate the derivatives.

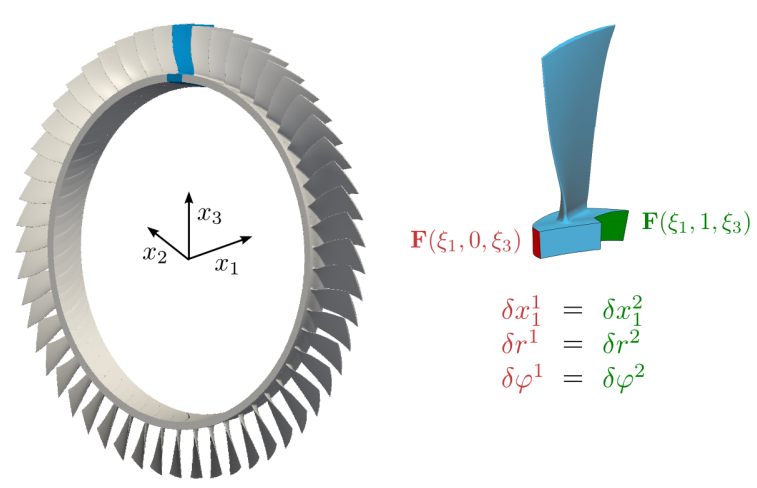

Figure 3: Left: The entire blisk is located in a Cartesian coordinate system with the $x_{1}$-axis as the axis of rotation and the $x_{3}$-axis as the radial direction of the blade to be considered (blue). Right: Cylindrical periodic boundary conditions of the displacements for the translational sides of the block.

\section{Simulations}

In this section we study the deformation of turbine blades under the assumption of linear elasticity by considering all major loads and boundary conditions arising from the structural mechanic simulation process in an industrial environment. We start by defining the setting of the blade simulation, especially the periodic boundary conditions, and proceed by applying successively the required mechanical and thermal loads.

\subsection{Setting}

We consider a blade $\boldsymbol{\Omega}$ as a periodically symmetric segment of a blisk (blade integrated disk) with respect to the $x_{1}$-axis as the axis of rotation, see Figure 3 . Therefore, it is required that the blade is deformed as a rotationally symmetric part of the blisk and the displacements on the translational sides of the block, $\mathbf{F}\left(\xi_{1}, 0, \xi_{3}\right)$ and $\mathbf{F}\left(\xi_{1}, 1, \xi_{3}\right)$ for $0 \leq \xi_{3} \leq \bar{\xi}_{3}$, have to be periodic in the cylindrical coordinate system $\left(x_{1}, r, \varphi\right)$,

$$
\begin{aligned}
x_{1} & =x_{1} \\
r & =\sqrt{x_{2}^{2}+x_{3}^{2}} \\
\varphi & =\arctan \frac{x_{3}}{x_{2}} .
\end{aligned}
$$

This implies the periodic boundary conditions for the displacements $u_{i}$,

$$
\begin{aligned}
u_{1}^{1} & =u_{1}^{2} \\
\frac{x_{2}^{1}}{r^{1}} u_{2}^{1}+\frac{x_{3}^{1}}{r^{1}} u_{3}^{1} & =\frac{x_{2}^{2}}{r^{2}} u_{2}^{2}+\frac{x_{3}^{2}}{r^{2}} u_{3}^{2} \\
\frac{x_{3}^{1}}{r^{1} r^{1}} u_{2}^{1}+\frac{x_{2}^{1}}{r^{1} r^{1}} u_{3}^{1} & =\frac{x_{3}^{2}}{r^{2} r^{2}} u_{2}^{2}+\frac{x_{2}^{2}}{r^{2} r^{2}} u_{3}^{2},
\end{aligned}
$$

where the superscript indicates the side of the block, see Figure 3. We relate the given constraints for the displacements $u_{i}$ to the control variables $q_{j}$ by the Greville abscissae of their basis functions.

We will compare the results of the isogeometric simulations with the numerical approximations obtained by the classical finite element method based on an automatically generated mesh (with quadratic hexahedral elements) of the blade described in Section 2 and the structural finite element program CalculiX [12].

\subsection{Centrifugal force}

The primary mechanical load acting on our geometry are the centrifugal forces caused by the rotation of the blisk. 


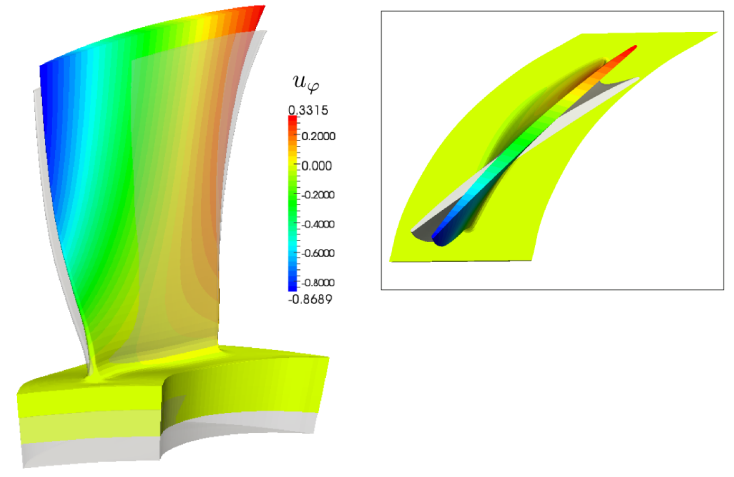

Figure 4: The figure shows the initial shape of the blade (transparent) and the deformed blade (colored according to the displacement component $u_{\varphi}$ ). The top view illustrates the twist of the airfoil.

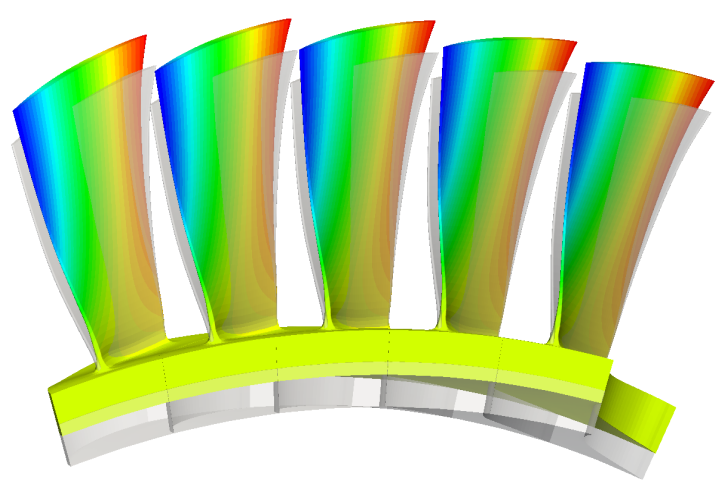

Figure 5: Deformation of a section of the blisk under the centrifugal force. The deformed blades are colored according to the displacement component $u_{\varphi}$.

For a given angular velocity $\omega$, the centrifugal force $\hat{\mathbf{b}}(\mathbf{x})$ of a point $\mathbf{x}=\left(x_{1}, x_{2}, x_{3}\right)^{T} \in \boldsymbol{\Omega}$ is defined by

$$
\hat{\mathbf{b}}(\mathbf{x})=\rho \omega^{2} \mathbf{r}(\mathbf{x}),
$$

for the density $\rho$ and the vector $\mathbf{r}(\mathbf{x})$ in radial direction. We ignore the surface pressure and thermal load in the following simulation examples and incorporate the discussed periodic boundary conditions for the block of the blade.

The centrifugal force causes a radial expansion of the blisk and a twist of the airfoils. Figure 4 visualizes the displacement component $u_{\varphi}$ and the blade deformation for the angular velocity $\omega=100$. The simulation of several blades of a blisk is shown in Figure 5 .

In general, a blisk consists of identical blades and by the incorporation of the periodic boundary conditions, the simulation of one blade can be transferred to the entire blisk. This reduces the computational effort for the simulation of several blades respectively the entire blisk significantly.

We compare the results of the isogeometric analysis and the finite element analysis in Figure 6 and 7 . The highest stress occurs at the transition part between the airfoil and the base of the blade as the so-called fillet. Consequently, the design of the fillet is one of the crucial steps in the design of turbine blades.

While the isogeometric method already produces excellent results for the initial parameterization defined by around 4000 control points, the finite element method requires at least 40000 nodes to obtain a competitive result.

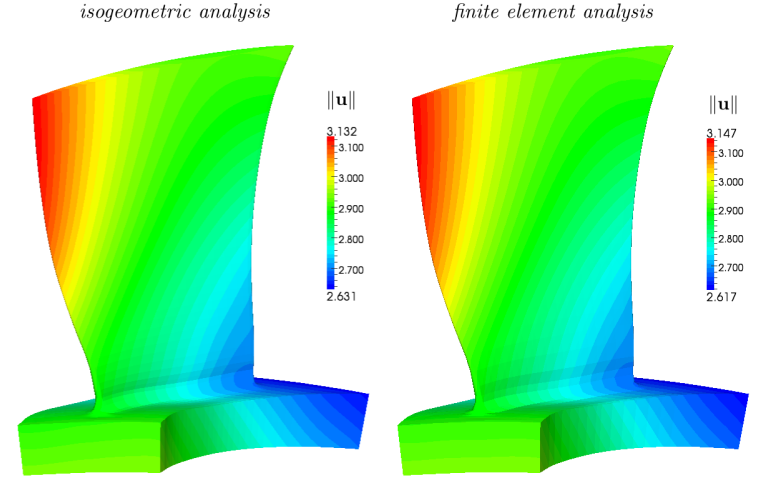

Figure 6: Analysis of the blade deformation under centrifugal forces with dof iga $=4004$ for the isogeometric method and $\operatorname{dof}_{\text {fea }}=43228$ for the finite element method.
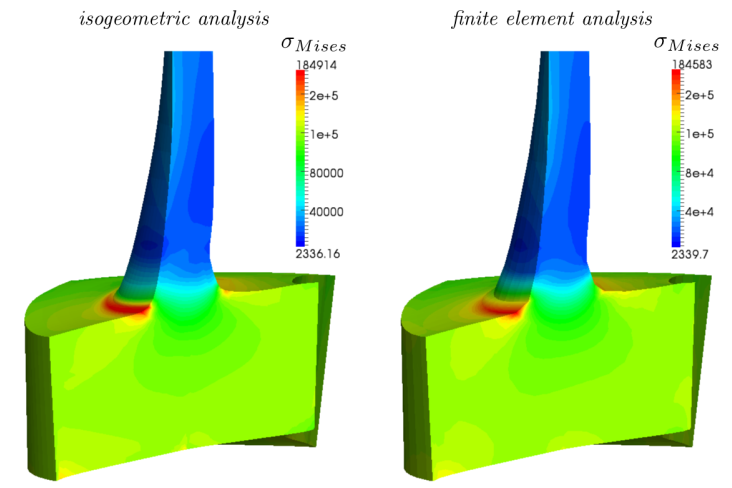

Figure 7: Analysis of the resulting stress by the von Mises stress $\sigma_{\text {Mises }}$ with dof iga $=4004$ and dof $_{\text {fea }}=43228$. The blade is sliced for a more detailed view into its interior.

The convergence behavior of the two methods for increasing numbers of degrees of freedom (i.e., increasingly find discretizations) is shown in Figure 8.

\subsection{Surface pressure}

The blades move through the air which is compressed or expanded according to the position of the blade within the engine. The shape of the airfoil generates a pressure gradient between the pressure side and the suction side accompanied by the velocity difference. The component of the resulting force perpendicular to the direction of motion is called lift.

The department of aerodynamics of MTU simulates the flow within the engine and provides a discretized pressure distribution on the surface of the blades, see Figure 9.

The following steps are performed in order to integrate the pressure distribution into our simulation process:

1. Four parametric boundary surfaces of the blade part $\mathbf{F}\left(\xi_{1}, \xi_{2}, \xi_{3} \geq \bar{\xi}_{3}\right)$ are identified by setting one of the parameters $\bar{\xi}_{1}, \xi_{2}$ to 0 or 1 .

2. The pressure distribution on the surface of the blade above the block $\left(\xi_{3} \geq \bar{\xi}_{3}\right)$, given by a point cloud $\mathbf{p}_{i}=\left(x_{1 i}, x_{2 i}, x_{3 i}, p_{i}\right) \in \mathbb{R}^{4}$ with the pressure $p_{i}$ and $\left(x_{1 i}, x_{2 i}, x_{3 i}\right)^{T} \in \boldsymbol{\Gamma}$, is parameterized with respect to their location on one of the four boundary surfaces.

3. An additional fourth component $d_{p j}$ of the control points $\mathbf{d}_{j}=\left(d_{1 j}, d_{2 j}, d_{3 j}, d_{p j}\right)$ of the boundary surfaces is calculated by approximating the onedimensional pressure data $p_{j}$ in a least-squares sense 


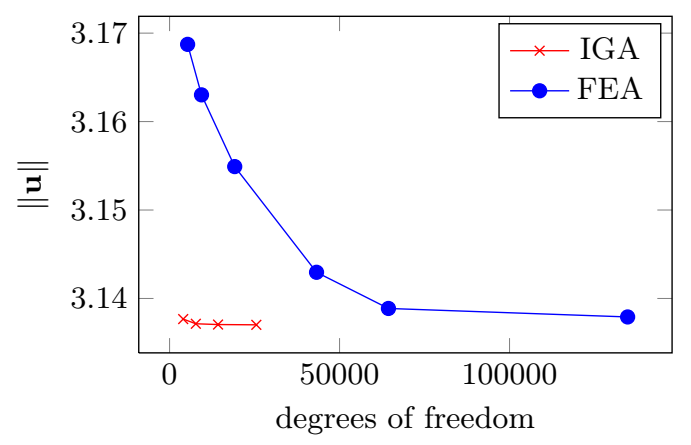

Figure 8: Displacement of the top corner at the leading edge of the blade under the centrifugal force with $\omega=100$.

using the generated parameterization. For example, the pressure component of the boundary surface $S_{p}\left(\xi_{1}, \xi_{3}\right)=\bar{N}_{j}\left(\xi_{1}, \xi_{3}\right) d_{p j}$ for $\xi_{2}=0$ is approximated by

$$
\sum_{i}\left\|S_{p}\left(\xi_{1 i}, \xi_{3 i}\right)-p_{i}\right\|^{2} \rightarrow \min _{d_{p j}}
$$

The B-spline basis is refined by inserting additional knots if the fitting error exceeds a user-defined tolerance. This leaves the geometry and parameterization of the blade unchanged.

Adding the pressure control variables to the related control points of the geometry function $\mathbf{F}$ defines a continuous pressure distribution on the blade surface. Furthermore we still have one representation of the simulation model and the B-spline basis functions have to be evaluated only once for the generation of the linear system (17).

For the following analysis of the blade deformation caused by the pressure gradient we ignore the centrifugal force. Figure 9 visualizes the fitted pressure distribution and the resulting deformation of the blade. Figure $10 \mathrm{com}-$ pares the displacement for the isogeometric method and the finite element method. The resulting stress for both methods is shown in Figure 11. In this case, the numbers of degrees of freedom for the finite element method required to obtain the same accuracy as IGA is even higher, due to the (piecewise quadratic) approximation of the pressure distribution. For each element face of the blade surface the pressure component of the geometry function is evaluated at the face nodes and scaled by the face area.

\subsection{Thermal expansion}

The compression and expansion of the air in the turbine leads to an inhomogeneous temperature distribution and consequently a temperature gradient within the blades. In this section we simulate the resulting thermal expansion of the blade.

The department of thermodynamics of MTU simulates the thermal conduction within the blades and provides a three-dimensional point cloud of the discretized temperature distribution which we incorporate in a natural way:

1. The temperature distribution within the blade, given by the point cloud $\mathbf{p}_{i}=\left(x_{1 i}, x_{2 i}, x_{3 i}, t_{i}\right) \in \mathbb{R}^{4}$ with the temperature $t_{i}$ and $\left(x_{1 i}, x_{2 i}, x_{3 i}\right)^{T} \in \boldsymbol{\Omega}$, is directly parameterized by the geometry function $\mathbf{F}\left(\xi_{1}, \xi_{2}, \xi_{3}\right)$.

2. An additional fifth component $d_{t j}$ of the control points $\mathbf{d}_{j}=\left(d_{1 j}, d_{2 j}, d_{3 j}, d_{p j}, d_{t j}\right)$ is calculated by approximating the temperature component $F_{t}\left(\xi_{1}, \xi_{2}, \xi_{3}\right)=\bar{N}_{j}\left(\xi_{1}, \xi_{2}, \xi_{3}\right) d_{t j}$ in a least-squares sense,

$$
\sum_{i}\left\|F_{t}\left(\xi_{1 i}, \xi_{2 i}, \xi_{3 i}\right)-t_{i}\right\|^{2} \rightarrow \min _{d_{t j}}
$$

We again use knot insertions of the given B-spline basis to increase the fitting quality, if required.

The extended geometry function represents a continuous function of the temperature, the pressure and the geometry which can be efficiently and stably evaluated. As a consequence of the inhomogeneous tempera-

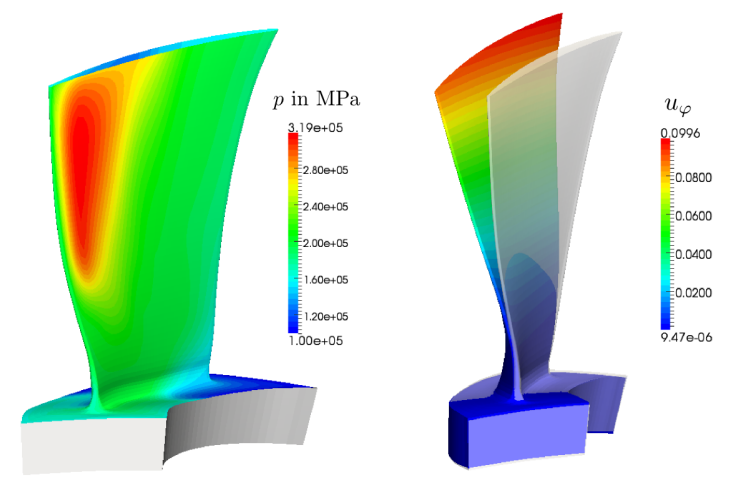

Figure 9: Left: The profile of the airfoil generates a pressure gradient between the high pressure at the pressure side and the lower pressure on the suction side. Right: The initial (transparent) and deformed shape of the blade (colored according to the displacement component $u_{\varphi}$ ).
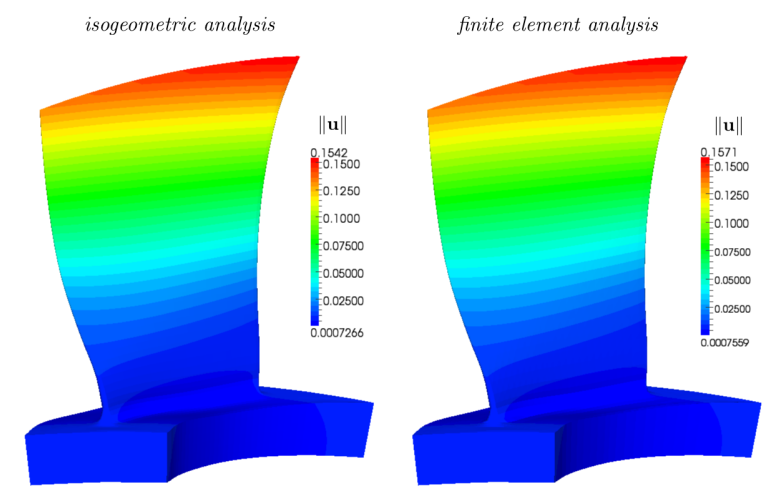

Figure 10: Analysis of the deformation caused by the surface pressure with dof iga $=8504$ and dof $_{\text {fea }}=96768$.

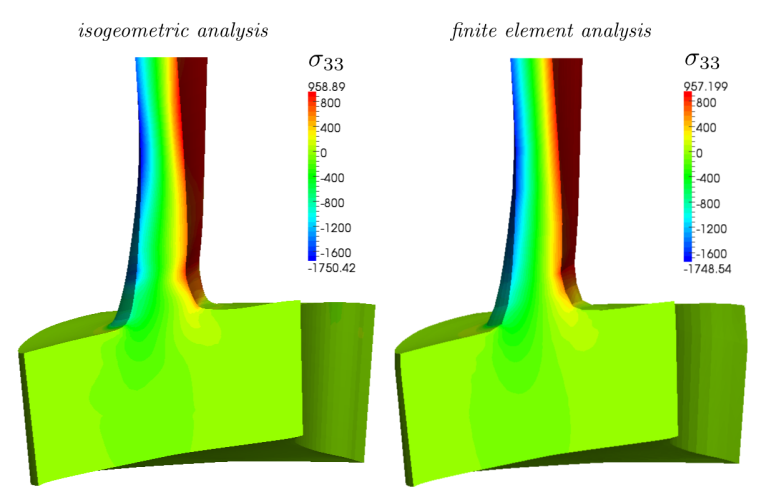

Figure 11: Analysis of the resulting stress by the normal stress $\sigma_{33}$ with dof iga $=8504$ and dof $\mathrm{f}_{\text {fea }}=96768$. The blade is sliced for a more detailed view into its interior.

ture, the Young's modulus $E\left(\xi_{1}, \xi_{2}, \xi_{3}\right)$ and the thermal 
expansion coefficient $\alpha\left(\xi_{1}, \xi_{2}, \xi_{3}\right)$ are no longer constant within the blade. We represent them by piecewise linear functions with respect to the temperature $T\left(\xi_{1}, \xi_{2}, \xi_{3}\right)$ for $\left(\xi_{1}, \xi_{2}, \xi_{3}\right)^{T} \in \boldsymbol{\Omega}_{0}$.

Figure 12 shows the temperature distribution and the resulting thermal expansions. The lowest temperature is located at the block and increases with the velocity of the flow in the middle of the flow channel. The resulting deformation is rather small and therefore we leave the surface pressure and centrifugal forces out of consideration (as they would hide the influence of the temperature otherwise). The number of nodes for an accurate finite element analysis is again very high as a consequence of the approximation of the temperature field. The resulting displacement field is visualized in Figure 13, the von Mises stress in Figure 14.

\section{Conclusions}

The numerical results reported in this paper demonstrate the capabilities of isogeometric analysis in a real-world industrial environment with challenging numerical simulation problems. More precisely, we studied the deformation of blades of aircraft engines under the influence of centrifugal forces, air pressure, and inhomogeneous temperature distribution.

It was shown that results comparable with the existing finite element technology can be obtained by using a much coarser discretization - typically, only $10 \%$ of the number of degrees of freedom are required. We feel these are impressive results, which clearly demonstrate the outstanding capability of the new method. Moreover, isogeometric analysis provides the added advantage of using an exact representation of the geometry simultaneously for numerical simulation and for geometric design, thus eliminating the need for conversion processes such as mesh generation.

The implementation of isogeometric solvers is still under development. In particular, there are currently only a few solvers available that can deal with three-dimensional simulation problems. The experimental solver available to us, which was developed in the frame of the EXCITING project, is limited to single patch geometric representations. We enhanced the capabilities of this solver by providing additional possibilities for specifying boundary conditions and material properties.

Clearly, the existing finite element solvers have undergone a long history of software development and efficiency improvements, and it would be too much to expect that isogeometric solvers can beat them from the start with respect to all performance measures, including overall computation timings (including the time needed to assemble the matrices). Based on our experience we are convinced that isogeometric solvers for multi-patch geometric representations will be able to compete successfully with the existing finite element technology not only with respect to the number of required degrees of freedom needed to achieve a certain accuracy, but also with respect to computing times. Further experiments have been shown that the computational complexity for the isogeometric and the finite element method are nearly the same, for more details see [15].

Besides software development, there are also several theoretical aspects that deserve further investigations. These include the use of adaptive refinement in isogeometric analysis and efficient techniques for evaluating the stiffness matrices, as well as automatic techniques for multipatch domain parameterization. These are interesting and challenging topics for further research.
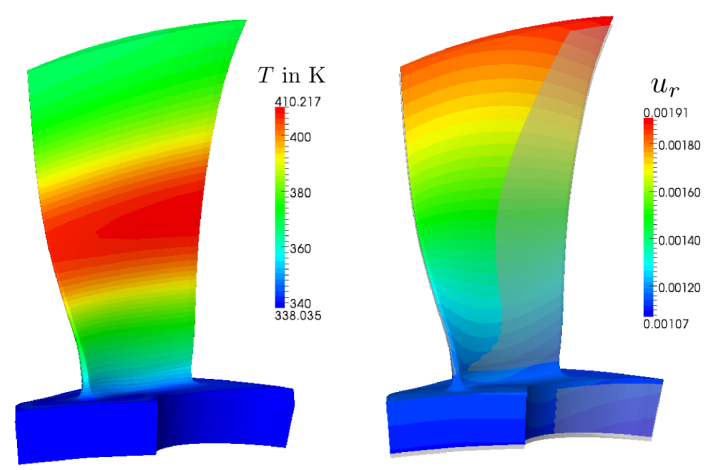

Figure 12: Left: The given temperature distribution of the blade. Right: The initial (transparent) and deformed shape of the blade (colored according to the radial displacement component $u_{r}$ ).
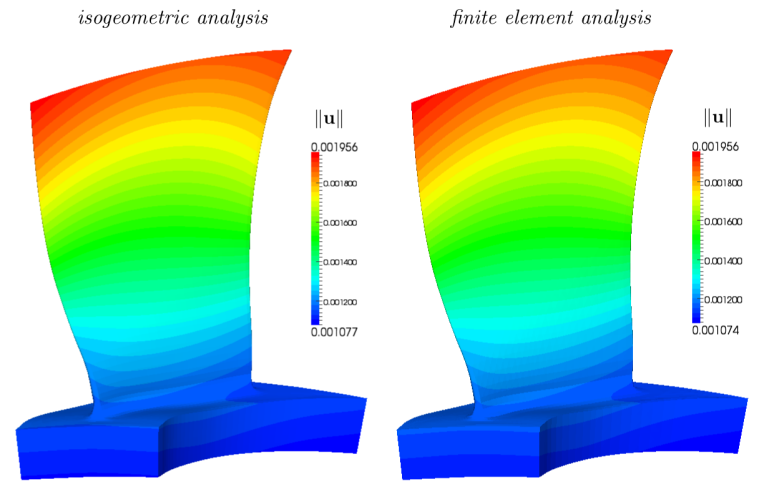

Figure 13: Analysis of the thermal expansion for the isogeometric and finite element method with dof iga $=4004$ and $\operatorname{dof}_{\mathrm{fea}}=64376$
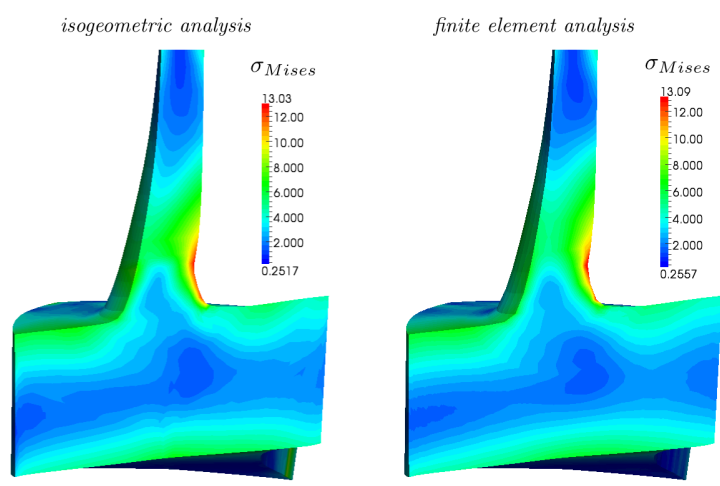

Figure 14: Analysis of the resulting stress by the von Mises stress $\sigma_{\text {Mises }}$ with dof iga $=4004$ and dof $_{\text {fea }}=64376$. The blade is sliced for a more detailed view into its interior.

\section{Acknowledgment}

B. Jüttler and A.-V. Vuong were supported by the European Union through the FP 7 project "Exact Geometry Simulation for Optimized Design of Vehicles and Vessels" (EXCITING, GA no. 218536).

\section{References}

[1] M. Aigner, C. Heinrich, B. Jüttler, E. Pilgerstorfer, B. Simeon, and A.-V. Vuong. Swept Volume Parameterization for Isogeometric Analysis. In Proc. IMA Int. Conf. Mathematics of Surfaces XIII, pages 19-44. Springer, 2009. 
[2] F. Auricchio, F. Calabro, T. J. R. Hughes, A. Reali, and G. Sangalli. A Simple Algorithm for Obtaining Nearly Optimal Quadrature Rules for NURBS-based Isogeometric Analysis. ICES Technical Report 12-04, The University of Texas at Austin, 2012.

[3] Y. Bazilevs, L. Beirão da Veiga, J. A. Cottrell, T. J. R. Hughes, and G. Sangalli. Isogeometric analysis: approximation, stability and error estimates for $h$-refined meshes. Math. Models Methods Appl. Sci., 16(7):1031-1090, 2006.

[4] Y. Bazilevs, V. M. Calo, J. A. Cottrell, J. A. Evans, T. J. R. Hughes, S. Lipton, M. A. Scott, and T. W. Sederberg. Isogeometric analysis using T-splines. Comput. Methods Appl. Mech. Engrg., 199(5-8):229-263, 2010.

[5] L. Beirão da Veiga, A. Buffa, J. Rivas, and G. Sangalli. Some estimates for h-p-k-refinement in isogeometric analysis. Numerische Mathematik, 118:271-305, 2005.

[6] L. Beirão da Veiga, A. Buffa, D. Cho, and G. Sangalli. IsoGeometric analysis using T-splines on two-patch geometries. Comput. Methods Appl. Mech. Engrg., 200(2122):1787-1803, 2011.

[7] A. F. Bower. Applied Mechanics of Solids. CRC Press, 2010.

[8] A. Buffa, G. Sangalli, and R. Vázquez. Isogeometric analysis in electromagnetics: B-splines approximation. Comput. Methods Appl. Mech. Engrg., 199(17-20):1143-1152, 2010.

[9] E. Cohen, T. Martin, R. Kirby, T. Lyche, and R. Riesenfeld. Analysis-aware modeling: Understanding quality considerations in modeling for isogeometric analysis. Comput. Methods Appl. Mech. Engrg., 199(5-8):334 - 356, 2010.

[10] J. Deng, F. Chen, and Y. Feng. Dimensions of spline spaces over T-meshes. J. Comp. Appl. Math., 194:267-283, 2006.

[11] J. Deng, F. Chen, X. Li, C. Hu, T. W., Z. Yang, and Y. Feng. Polynomial splines over hierarchical T-meshes. Graphical Models, 70:76-86, 2008.

[12] G. Dhondt. The Finite Element Method for ThreeDimensional Thermomechanical Applications. Wiley and Sons, 2004. http://www.calculix.de.

[13] M. R. Dörfel, B. Jüttler, and B. Simeon. Adaptive isogeometric analysis by local $h$-refinement with T-splines. Comput. Methods Appl. Mech. Engrg., 199(5-8):264-275, 2010.

[14] T. Elguedj, Y. Bazilevs, V. Calo, and T. Hughes. $\bar{b}$ and $\bar{f}$ projection methods for nearly incompressible linear and nonlinear elasticity and plasticity using higher-order NURBS elements. Comput. Methods Appl. Mech. Eng., 197(33-40):2732-2762, 2008.

[15] D. Großmann. Volumetric Geometry Reconstruction and Isogeometric Simulation of Turbine Blades for Aircraft Engines. PhD thesis, JKU Linz, 2012.

[16] D. Großmann and B. Jüttler. Volumetric geometry reconstruction of turbine blades for aircraft engines. In J.-D. Boissonnat, P. Chenin, A. Cohen, C. Gout, T. Lyche, M.-L. Mazure, and L. Schumaker, editors, Curves and Surfaces, volume 6920 of Lecture Notes in Computer Science, pages 280-295. Springer Berlin / Heidelberg, 2012.

[17] J. Hoschek and D. Lasser. Fundamentals of computer aided geometric design. A.K. Peters, 1996.

[18] T. Hughes. The Finite Element Method: Linear Static and Dynamic Finite Element Analysis. Prentice Hall, Englewood Cliffs, 1987.

[19] T. Hughes, J. Cottrell, and Y. Bazilevs. Isogeometric analysis: CAD, finite elements, NURBS, exact geometry and mesh refinement. Comput. Methods Appl. Mech. Engrg., 194(39-41):4135-4195, 2005.

[20] T. Hughes, J. Cottrell, and Y. Bazilevs. Isogeometric Analysis: Toward Integration of CAD and FEA. Wiley, Chichester, 2009.

[21] T. Hughes, A. Reali, and G. Sangalli. Efficient quadrature for NURBS-based isogeometric analysis. Comput. Methods Appl. Mech. Engrg., 199(5-8):301-313, 2010.
[22] T. J. R. Hughes, A. Reali, and G. Sangalli. Efficient quadrature for NURBS-based isogeometric analysis. Comput. Methods Appl. Mech. Engrg., 199(5-8):301-313, 2010.

[23] X. Li, J. Deng, and F. Chen. Polynomial splines over general T-meshes. The Visual Computer, 26:277-286, 2010.

[24] T. Martin, E. Cohen, and R. M. Kirby. Volumetric parameterization and trivariate B-spline fitting using harmonic functions. Comput. Aided Geom. Design, 26(6):648-664, 2009.

[25] N. Nguyen-Thanh, H. Nguyen-Xuan, S. P. A. Bordas, and T. Rabczuk. Isogeometric analysis using polynomial splines over hierarchical T-meshes for two-dimensional elastic solids. Comput. Methods Appl. Mech. Engrg., 200(21-22):1892-1908, 2011.

[26] P. N. Nielsen, A. R. Gersborg, J. Gravesen, and N. L. Pedersen. Discretizations in isogeometric analysis of NavierStokes flow. Comput. Methods Appl. Mech. Engrg., 200(4546):3242-3253, 2011.

[27] M. A. Scott, X. Li, T. W. Sederberg, and T. J. R. Hughes. Local refinement of analysis-suitable T-splines. Comput. Methods Appl. Mech. Engrg., 213-216:206-222, 2012.

[28] T. Takacs and B. Jüttler. Existence of stiffness matrix integrals for singularly parameterized domains in isogeometric analysis. Comput. Methods Appl. Mech. Engrg., 200:35683582, 2011.

[29] R. L. Taylor. Isogeometric analysis of nearly incompressible solids. International Journal for Numerical Methods in Engineering, 87(1-5):273-288, 2011.

[30] A.-V. Vuong, C. Giannelli, B. Jüttler, and B. Simeon. A hierarchical approach to adaptive local refinement in isogeometric analysis. Comput. Methods Appl. Mech. Engrg., 200(49-52):3554-3567, 2011.

[31] K. Wang, X. Li, B. Li, H. Xu, and H. Qin. Restricted trivariate polycube splines for volumetric data modeling. IEEE Trans. Vis. Comp. Graphics, 2011. DOI 10.1109/TVCG.2011.102. 